NOTA TECNICA

\title{
Impacto de la duración y geometría del control de malezas en la productividad de plantaciones de Pinus radiata D. Don en cinco ecosistemas del sur de Chile*
}

\author{
The impact of duration and geometry of weed control in productivity of Pinus radiata \\ plantations in five southern ecosystems of Chile
}

\author{
JOSE ALVAREZ ${ }^{1}$, RODRIGO VENEGAS ${ }^{2}$, CAROLINA PEREZ ${ }^{3}$ \\ ${ }^{1}$ Dpto. Productividad de Sitios, Forestal Mininco S.A. Casilla 43-C Concepción, Chile. \\ E-mail: jalvarez@formin.cmpc.cl \\ ${ }^{2}$ Dpto. Productividad de Sitios, Forestal Mininco S.A., Los Angeles, Chile. E-mail: rvenegas@formin.cmpc.cl \\ 3Ingeniero Agrónomo. Temuco, Chile. E-mail: cperezcobar@hotmail.com
}

\begin{abstract}
SUMMARY
The effect of weed control on the initial plantation productivity of Pinus radiata D. Don was investigated in five representative southern ecosystems of Chile. Different periods without interference $(0,0+1,0+1+2)$ and the geometry of control (total, band, spot, nil) were evaluated during the first four to six years after establishment. The best results for volume were total and band weed control. The volumes did not differ for granitic (alfisol) and metamorphic soils (ultisol) from one to three years without interference. In sandy soil (entisol), three periods with weed control were best, and in volcanic ash soils (andisols) there were no differences between one and three periods weed free. In red clay soils (alfisols) the best strategy was two periods weed-free. The economic analysis suggests site specific prescriptions: one control over the total surface in sandy soil, two years of band control in more humid ecosystems or those with high productivity potential. Recommendations and future lines of work in this field are discussed.
\end{abstract}

Key words: Pinus radiata, weed control, productivity.

\section{RESUMEN}

Se estudió el efecto del control de malezas en la productividad de plantaciones de Pinus radiata D. Don, en 5 ecosistemas representativos del sur de Chile. En cada sitio se evaluaron distintos períodos con control de malezas $(0,0+1,0+1+2)$ y geometría de control (Total, Banda $2 \mathrm{~m}$ ancho, Taza $1 \mathrm{~m}^{2}$, Sin control), durante los primeros 4-6 años de establecimiento. Los resultados en volumen señalan que los mejores tratamientos de control fueron Total y Banda. En los suelos graníticos (Alfisol) y metamórficos costeros (Ultisol) no se encontraron diferencias significativas entre 1 a 3 períodos con control de malezas. En arenas secas (Entisol), 3 períodos fueron significativamente superiores y en sitios de cenizas volcánicas recientes (Andisol) no hubo diferencias entre 1 a 3 períodos libre de malezas. En suelos rojo arcillosos (Alfisol) la mejor estrategia es 2 períodos de control de malezas. El análisis económico permite sugerir prescripciones sitio específico, desde 1 control a superficie total en sitios de arenas secas y 2 controles con banda de $2 \mathrm{~m}$ de ancho, en ecosistemas más húmedos o de mayor productividad. Las recomendaciones y líneas de trabajo futuro en este campo son analizadas.

Palabras clave: Pinus radiata, control de malezas, productividad.

* El estudio forma parte del Programa de Desarrollo en Productividad de Sitios de Forestal Mininco S.A. 
BOSQUE 25(2): 57-67, 2004

Impacto de la duración y geometría del control de malezas en la productividad de plantaciones...

\section{INTRODUCCION}

La presencia de malezas en el ecosistema forestal condiciona la acción de numerosos factores, algunos favorables; como la protección del suelo contra procesos erosivos y otros negativos como pérdida de crecimiento de cultivos, plagas, etc. Kogan y Figueroa (1) e Izquierdo (2) señalan que la presencia de las malezas afecta negativamente la sobrevivencia y el crecimiento de una plantación forestal. Por su parte, Adams y Dutkowsky (3) indican que la competencia de malezas en suelos húmedos durante el primer período estival, después de la plantación, puede tener efectos severos en el crecimiento inicial del Pinus radiata D. Don debido a las diferentes formas de interferencia con las especies forestales, lo cual puede llegar a producir una significativa supresión del crecimiento del cultivo y hasta una extendida mortalidad (4). Al mismo tiempo, el estrés causado por la interferencia de malezas puede incrementar la susceptibilidad al ataque de plagas y enfermedades, además, Marsico (5), citado por Montenegro (6), indica que estas malezas muchas veces son reservorios de agentes patógenos.

Incrementar el crecimiento, en respuesta al control de malezas, es usualmente explicado en términos de un mejor acceso de las especies arbóreas al agua (7) o nutrientes (4, 8-10). Adams et al. (4) señalan que esta ventaja de incrementar la actividad fisiológica de los árboles es primordial para el incremento del área foliar, mayor intercepción de luz y actividad fotosintética para el cultivo, lo que, según Kogan y Figueroa (1) y Montenegro (6), se traduce en una mayor supervivencia de las plantas de pino y también en notables incrementos en la producción de biomasa. Al respecto, MacLaren (11), citado por Bisognin (12), reportó que en plantaciones de Pinus radiata D. Don de once años de edad se presentó un incremento de $50 \%$ de crecimiento en volumen entre árboles que crecían con control de malezas sobre los que crecían sin control. Dado lo anterior, cabe destacar la importancia económica del control de malezas, el cual afecta a la producción forestal en un porcentaje variable de acuerdo a: el ecosistema, la intensidad del control de malezas y la duración del mismo. Surge entonces la necesidad de realizar estudios en esta área con el fin de poder conocer la intensidad (geometría) y el tiempo (años) por el cual se debe controlar la vegetación existen- te. El objetivo principal de esta investigación es determinar, para distintos sitios, la intensidad y periodicidad óptima de control de malezas para lograr el máximo crecimiento de Pinus radiata $\mathrm{D}$. Don.

\section{MATERIAL Y METODOS}

Descripción de sitios. La descripción de los sitios experimentales se puede apreciar en el cuadro 1 . Las zonas en que estos sitios se ubican poseen los siguientes climas: San Pedro ( $\left.37^{\circ} 35^{\prime}, 72^{\circ} 22^{\prime}\right)$, clima templado mesotermal estenotérmico, Escuadrón $\left(36^{\circ} 56^{\prime}, 73^{\circ} 8^{\prime}\right)$, Etiopía $\left(37^{\circ} 54^{\prime}, 72^{\circ} 22^{\prime}\right)$ y Entrecaminos $\left(38^{\circ} 28^{\prime}, 72^{\circ} 13^{\prime}\right)$, clima templado mesotermal inferior estenotérmico mediterráneo subhúmedo y Coihueco $\left(37^{\circ} 28^{\prime}, 72^{\circ} 3^{\prime}\right)$, clima templado mesotermal inferior estenotérmico mediterráneo semiárido (anexo 1). (13)

Silvicultura. Respecto a la preparación de suelos en San Pedro y Escuadrón se realizó casilla mecanizada con excavadora (1.000 casillas/ha), en Etiopía y Coihueco se efectuó un subsolado con bulldozer más rastra Savannah $(80 \mathrm{~cm}$ de profundidad) y en Entrecaminos se realizó preparación de suelo con sólo subsolado agrícola (40 $\mathrm{cm}$ profundidad). En la fertilización se empleó en cada fundo una mezcla de $10 \mathrm{~g} / \mathrm{pl} \mathrm{N}, 50 \mathrm{~g} / \mathrm{pl}_{2} \mathrm{O}_{5}$ y $3 \mathrm{~g} / \mathrm{pl}$ B. En cuanto al control de malezas se emplearon en preplantación: 4 1/ha de Glifosato, al Año 0: Hexazinona $(2,5 \mathrm{~kg} / \mathrm{ha}$ ) año 1 y Glifosato $(3 \mathrm{l} / \mathrm{ha})$ + Atrazina (4 kg/ha) al año 2. Lo anterior es válido para todos los fundos con excepción de Coihueco donde se utilizaron en preplantación: 4 1/ha de Glifosato, al año 0: Atrazina (4 kg/ha) + Glifosato (3 1/ha) al año 1 y 2.

Las plantas utilizadas para cada ensayo correspondieron al mejor material genético disponible por sitio, el que fue asignado según la política de mejoramiento genético de Forestal Mininco. Asimismo, las plantas fueron seleccionadas en cuanto a DAC (diámetro a la altura del cuello = DAC) y altura inicial, de manera tal de contar con material homogéneo. Lo anterior se verificó mediante el test de homogeneidad de varianzas de Bartlet (14).

Diseño experimental y tratamientos. El diseño experimental de los ensayos corresponde a parcelas divididas en bloques completamente al azar 


\section{CUADRO 1}

Descripción de los sitios experimentales Description of the experimental sites.

\begin{tabular}{|c|c|c|c|c|c|}
\hline $\begin{array}{c}\text { Sitios } \\
\text { Experimentales }\end{array}$ & Ecosistemas & $\begin{array}{l}\text { Serie de } \\
\text { Suelo }\end{array}$ & $\begin{array}{l}\text { Tipo de } \\
\text { Suelo }\end{array}$ & $\begin{array}{l}\text { Fecha de } \\
\text { Inicio }\end{array}$ & $\begin{array}{c}\text { Principales } \\
\text { malezas presentes }\end{array}$ \\
\hline Escuadrón & $\begin{array}{l}\text { Cordillera de la } \\
\text { Costa y faldeos } \\
\text { occidentales }\end{array}$ & Pileo & $\begin{array}{c}\text { Metamórfico } \\
\text { costero } \\
\text { (Ultisol) }\end{array}$ & 1996 & $\begin{array}{l}\text { Vinagrillo, } \\
\text { sonchus, chépica }\end{array}$ \\
\hline San Pedro & Secano Interior & Cauquenes & $\begin{array}{l}\text { Granítico } \\
\text { (Alfisol) }\end{array}$ & 1996 & $\begin{array}{c}\text { Siete venas, } \\
\text { vinagrillo, cardo }\end{array}$ \\
\hline Etiopía & $\begin{array}{c}\text { Precordillera y } \\
\text { sectores abrigados } \\
\text { del valle Central }\end{array}$ & Collipulli & $\begin{array}{l}\text { Rojo arcilloso } \\
\text { (Alfisol) }\end{array}$ & 1998 & $\begin{array}{c}\text { Pasto cebolla, } \\
\text { vinagrillo, avenilla }\end{array}$ \\
\hline Entrecaminos & Precordillera & Santa Bárbara & $\begin{array}{c}\text { Trumao, cenizas } \\
\text { volcánicas recientes } \\
\text { (Andisol) }\end{array}$ & 1998 & $\begin{array}{l}\text { Hierba azul, } \\
\text { vinagrillo }\end{array}$ \\
\hline Coihueco & Valle Central & Coreo & $\begin{array}{l}\text { Arenales } \\
\text { (Entisol) }\end{array}$ & 1998 & $\begin{array}{l}\text { Hierba del paño, } \\
\text { vinagrillo, chépica }\end{array}$ \\
\hline
\end{tabular}

con tres repeticiones. En cada subparcela se consideraron 3 hileras de 10 plantas cada una. La descripción de los tratamientos se describe a continuación:

$\begin{array}{lll}\text { Tratamientos: } & - \text { Testigo } & \text { (T1) } \\ \text { Intensidad } & - \text { Total } & \text { (T2) } \\ & - \text { Banda } 2 \mathrm{~m} & \text { (T3) } \\ & - \text { Taza } 1 \mathrm{~m}^{2} & \text { (T4) } \\ \text { Subtratamientos: } & - \text { Año } 0 \\ \text { Periodicidad } & - \text { Año 0+1 } & \\ & - \text { Año } 0+1+2 & \end{array}$

Al momento de la evaluación, las plantas tenían entre 4 a 6 años de edad. Se consideraron las variables: DAC, DAP (diámetro a la altura del pecho $=$ DAP) y altura (h) como variables de medición y volumen como variable de respuesta, obtenido de acuerdo a la siguiente expresión [1]:

$$
\text { Volumen }\left(m^{3}\right)=A B \times h \times 0,25
$$

Donde:

$\mathrm{AB}=$ Area basal $\left(\mathrm{m}^{2}\right)$
$\mathrm{h}=$ Altura $(\mathrm{m})$
El análisis de varianza se realizó en base al modelo mixto Bifactorial con efecto jerárquico en parcelas divididas en bloques completamente al azar (S.P.S.S 8.0) ${ }^{1}$, cuyo modelo es:

$Y i j k=\mu+B k+\alpha i+\delta i k+\beta j+(\alpha \beta) i j+E i j k[2]$

donde, $\mu$ representa a la media general, $B k$ corresponde al efecto del bloque $\mathrm{k}$, $\alpha i$ representa el efecto del nivel i del factor $\alpha$, $\delta i k$ es el error experimental correspondiente al diseño de bloques completamente al azar (forma en que fueron equipadas las parcelas principales), $\beta j$ corresponde al efecto del nivel $\mathrm{j}$ del factor $\beta$, $(\alpha \beta) i j$ representa el efecto del tratamiento ij y Eijk es la variabilidad natural entre las subparcelas.

Para la comparación múltiple de promedios se utilizaron pruebas de Tukey al 5\% de significancia. En todos los ensayos se hizo un análisis del volumen por árbol.

La base del análisis económico utilizó las mediciones a los 4 y 6 años, donde la diferencia en altura y área basal, respecto al testigo, permitió estimar la ganancia de cada tratamiento. Como base

1 Copyright $(C) 2003$, SPSS Inc. 
BOSQUE 25(2): 57-67, 2004

Impacto de la duración y geometría del control de malezas en la productividad de plantaciones...

de comparación se utilizó el tratamiento en taza de $1 \mathrm{~m}^{2}$ de superficie, dado que este es un tratamiento más probable en términos de rusticidad. La ganancia volumétrica a la edad de 20 años se estimó en base a la misma ganancia en altura obtenida a la edad temprana, asumiendo una respuesta del tipo B según Snowdon y Waring (15). La proyección de ganancia volumétrica a la edad de 20 años se obtuvo mediante el uso de la versión 5.4 del Modelo Radiata ${ }^{2}$. La comparación del volumen obtenido en cada tratamiento permitió estimar la reducción de la edad de rotación. Asimismo, la ganancia obtenida permitió determinar el precio promedio de la nueva canasta de productos (trozos podados, nudosos y pulpables). Con los datos disponibles se pudo obtener el VPN (Valor Presente Neto) y la TIR (Tasa Interna de Retorno) diferencial de cada tratamiento, en comparación al control en taza de $1 \mathrm{~m}^{2}$. La tasa de descuento utilizada fue de $10 \%$ y la tasa de impuesto de $17 \%$.

\section{RESULTADOS Y DISCUSION}

Al analizar lo sucedido a los 4 años con los parámetros de medición, DAC y altura (cuadro 2), se evidencia superioridad del sitio metamórfico costero (Escuadrón), por las mejores condiciones de este sitio para el crecimiento inicial de Pinus radiata $\mathrm{D}$. Don.

En general, después de 2 a 4 años, es posible apreciar que el crecimiento en DAC y altura fue significativamente mayor donde se realizó control total y en banda por 2 a 3 años, excepto en los fundos Escuadrón y Coihueco, donde el control total fue estadísticamente superior a banda. El resultado anterior concuerda con lo obtenido por Richardson et al. (16), y además coincide con lo descrito por Adams y Dutkowski (3) donde una investigación experimental de la respuesta del crecimiento de Pinus radiata D. Don a diferentes patrones de control de malezas demostró que el crecimiento inicial de éstos, en el sur de Australia, es maximizado por aplicaciones de control total durante las dos primeras estaciones de crecimiento. Bisognin (12) señala en cambio, que a pesar de no haber diferencias significativas entre el control

2 (C) Modelo Agregado. Proyecto Modelo Nacional de Simulación de Pinus radiata D. Don. Fundación Chile. en banda y el control total, para las especies Pinus taeda y Pinus elliottii, el mejor tratamiento sería un control en banda por dos años.

No obstante, desde un punto de vista práctico, Richardson et al. (16) reportan que el mejor tratamiento en cuanto a costo efectivo y aceptable ambientalmente varía con el tipo de suelo local, clima y especies competidoras.

Cabe destacar la escasa ganancia en el sitio Entrecaminos, observada en el crecimiento de los árboles después de un año de plantación en el tratamiento control total de malezas. El resultado más eficiente del control del año 0 puede haberse debido a la excesiva sequía del año 1998. Lo anterior, coincide con lo reportado por Richardson et al. (16), quien sugiere que otros factores limitaron el crecimiento de éstos.

Respecto al control en tazas, Adams y Dutkowski (3) indican que no debería ser utilizado, excepto donde la erosión sea un problema. En este estudio se aprecia que el tratamiento en tazas es sólo superior al testigo, llegando a ser en algunos casos similar a éste (figuras 1, 2 y 3), no reportando mayores ganancias de crecimiento. Smethurst y Nambiar (9) señalan que la presencia de malezas en plantaciones jóvenes de Pinus radiata $\mathrm{D}$. Don pueden retardar ampliamente el crecimiento al competir por $\mathrm{N}$ mineral. El crecimiento de los árboles será mejorado manteniendo la plantación libre de malezas y añadiendo fertilizante nitrogenado, aunque altos suministros de $\mathrm{N}$ pueden inducir deformaciones al tronco. No obstante señalan que el control parcial de malezas puede beneficiar la productividad a largo plazo porque las malezas reducen la pérdida de $\mathrm{N}$.

Al evaluar DAP y altura, a los 4 a 6 años de edad (cuadro 2), se evidencia una situación más o menos similar a la descrita anteriormente; sin embargo, se visualizan divergencias al analizar la intensidad en la variable altura en los sitios San Pedro y Etiopía.

El volumen (4-6 años) sigue una tendencia similar a la ocurrida con las variables de medición, DAC y altura, en que los tratamientos total y banda son estadísticamente iguales y superiores, excepto en el sitio Etiopía, donde el mejor tratamiento es el control en banda. Al respecto, experiencias neozelandesas con Pinus radiata D. Don señalan que, en términos de costo, el método más efectivo es controlar malezas en bandas (2), aunque por la naturaleza del terreno no siempre es factible de aplicar. 
BOSQUE 25(2): 57-67, 2004

Impacto de la duración y geometría del control de malezas en la productividad de plantaciones...

CUADRO 2

Resultados de crecimiento y sobrevivencia de los ensayos entre 2 a 6 años de edad.

Two to six years' results for growth and survival trials.

\begin{tabular}{|c|c|c|c|c|c|c|c|c|c|c|c|c|c|c|c|c|c|}
\hline Fundo/Sitio & $\begin{array}{l}\text { Tratamiento } \\
\text { (Intensidad) }\end{array}$ & $\begin{array}{c}\text { Subtratamiento } \\
\text { (Periodicidad) }\end{array}$ & Edad & $\mathrm{DAC}$ & $(\mathrm{m}$ & & Alt & ra ( & & Edad & $\begin{array}{c}\text { Sobreviv. } \\
(\%)\end{array}$ & DAP & $(\mathrm{mr}$ & m) & & $\operatorname{ara}($ & \\
\hline & Sin control & Sin control & 4 & 98 & & $\mathrm{C}$ & 4,3 & & $\mathrm{~B}$ & 6 & 93 & 142,3 & & $\mathrm{~B}$ & 9,7 & $\mathrm{~B}$ & \\
\hline & & 0 & 4 & 121 & $\mathrm{c}$ & & 5,4 & $\mathrm{~b}$ & & 6 & 98 & 153,8 & & & 10,5 & $\mathrm{~b}$ & \\
\hline Escuadrón & Total & $0+1$ & 4 & 154 & $\mathrm{~b}$ & A & 6,4 & $\mathrm{a}$ & A & 6 & 99 & 168,6 & $a b$ & A & 11,6 & $\mathrm{a}$ & A \\
\hline (Metamórfico & & $0+1+2$ & 4 & 166,6 & $\mathrm{a}$ & & 6,3 & $\mathrm{a}$ & & 6 & 93 & 175,4 & $\mathrm{a}$ & & 11,4 & $\mathrm{a}$ & \\
\hline costero) & & 0 & 4 & 119,6 & $\mathrm{c}$ & & 5,3 & $\mathrm{~b}$ & & 6 & 94 & 157,5 & & & 10,6 & $\mathrm{~b}$ & \\
\hline & Banda $2 \mathrm{~m}$ & $0+1$ & 4 & 134 & $\mathrm{~b}$ & B & 5,7 & $\mathrm{a}$ & A & 6 & 97 & 158,7 & $a b$ & A & 11,1 & $\mathrm{a}$ & A \\
\hline (1996-2000) & & $0+1+2$ & 4 & 148 & $\mathrm{a}$ & & 6,1 & $\mathrm{a}$ & & 6 & 91 & 173,3 & & & 11,7 & $\mathrm{a}$ & \\
\hline & & 0 & 4 & 86,6 & $\mathrm{c}$ & & 3,8 & $\mathrm{~b}$ & & 6 & 96 & 129,8 & & & 9,0 & $\mathrm{~b}$ & \\
\hline & Taza $1 \mathrm{~m}^{2}$ & $0+1$ & 4 & 100,3 & $\mathrm{~b}$ & $\mathrm{C}$ & 4,1 & $\mathrm{a}$ & B & 6 & 94 & 140,1 & $a b$ & B & 9,4 & $\mathrm{a}$ & B \\
\hline & & $0+1+2$ & 4 & 105,3 & a & & 4,2 & $\mathrm{a}$ & & 6 & 92 & 143,6 & & & 9,7 & $\mathrm{a}$ & \\
\hline & Sin control & Sin control & 4 & 45,6 & $\mathrm{C}$ & & 1,9 & $\mathrm{~B}$ & & 5 & 86 & 45,3 & & $\mathrm{C}$ & 3,2 & $\mathrm{~B}$ & \\
\hline & & 0 & 4 & 57,3 & $\mathrm{~b}$ & & 2,3 & $\mathrm{a}$ & & 5 & 80 & 54 & & & 3,8 & $\mathrm{~b}$ & \\
\hline & Total & $0+1$ & 4 & 87,6 & $\mathrm{a}$ & A & 3,4 & $\mathrm{a}$ & A & 5 & 84 & 87,3 & $\mathrm{a}$ & A & 5,4 & $\mathrm{a}$ & A \\
\hline San Pedro & & $0+1+2$ & 4 & 92,3 & $\mathrm{a}$ & & 3,5 & $\mathrm{a}$ & & 5 & 83 & 87,7 & $\mathrm{a}$ & & 5,4 & $\mathrm{a}$ & \\
\hline (Granítico) & & 0 & 4 & 63,6 & $\mathrm{~b}$ & & 2,7 & $\mathrm{a}$ & & 5 & 77 & 59,7 & $\mathrm{~b}$ & & 4,1 & $\mathrm{~b}$ & \\
\hline & Banda $2 \mathrm{~m}$ & $0+1$ & 4 & 84 & $\mathrm{a}$ & A & 3,6 & $\mathrm{a}$ & A & 5 & 88 & 81,3 & a $A$ & $\mathrm{AB}$ & 5,3 & $\mathrm{a}$ & A \\
\hline (1996-2000) & & $0+1+2$ & 4 & 79,3 & $\mathrm{a}$ & & 3,0 & $\mathrm{a}$ & & 5 & 83 & 76,7 & $\mathrm{a}$ & & 5,0 & $\mathrm{a}$ & \\
\hline & & 0 & 4 & 55 & $\mathrm{~b}$ & & 2,3 & $\mathrm{a}$ & & 5 & 79 & 55,7 & $\mathrm{~b}$ & & 3,7 & $\mathrm{~b}$ & \\
\hline & Taza $1 \mathrm{~m}^{2}$ & $0+1$ & 4 & 60,6 & $\mathrm{a}$ & B & 2,4 & $\mathrm{a}$ & B & 5 & 76 & 63 & a $\mathrm{E}$ & $\mathrm{BC}$ & 4,2 & $\mathrm{a}$ & A \\
\hline & & $0+1+2$ & 4 & 60,6 & a & & 2,5 & $\mathrm{a}$ & & 5 & 84 & 57 & $\mathrm{a}$ & & $4,1 \mathrm{a}$ & & \\
\hline & Sin control & Sin control & 4 & 56,5 & $\mathrm{D}$ & & 3,0 & & D & 4 & 90 & 31,6 & & $\mathrm{D}$ & 3,0 & D & \\
\hline & & 0 & 4 & 84,8 & $\mathrm{c}$ & & 3,5 & $\mathrm{~b}$ & & 4 & 93 & 49,8 & $\mathrm{~b}$ & & 3,5 & $\mathrm{~b}$ & \\
\hline & Total & $0+1$ & 4 & 95,7 & $\mathrm{~b}$ & A & 3,6 & $\mathrm{~b}$ & A & 4 & 82 & 52,7 & $\mathrm{~b}$ & A & 3,6 & $\mathrm{~b}$ & A \\
\hline Coihueco & & $0+1+2$ & 4 & 98,3 & $\mathrm{a}$ & & 3,8 & $\mathrm{a}$ & & 4 & 92 & 56,6 & $\mathrm{a}$ & & 3,8 & $\mathrm{a}$ & \\
\hline (Arena) & & 0 & 4 & 75,7 & $\mathrm{c}$ & & 3,3 & $\mathrm{~b}$ & & 4 & 86 & 44,3 & $\mathrm{~b}$ & & 3,3 & $\mathrm{~b}$ & \\
\hline (2) & Banda $2 \mathrm{~m}$ & $0+1$ & 4 & 83,4 & $\mathrm{~b}$ & B & 3,4 & $\mathrm{~b}$ & B & 4 & 93 & 47,1 & $\mathrm{~b}$ & B & 3,4 & $\mathrm{~b}$ & B \\
\hline (1998-2002) & & $0+1+2$ & 4 & 89,1 & $\mathrm{a}$ & & 3,7 & $\mathrm{a}$ & & 4 & 93 & 53,5 & $\mathrm{a}$ & & 3,7 & $\mathrm{a}$ & \\
\hline & & 0 & 4 & 67,9 & $\mathrm{c}$ & & 3,0 & $\mathrm{~b}$ & & 4 & 90 & 38,3 & $\mathrm{~b}$ & & 3,0 & $\mathrm{~b}$ & \\
\hline & Taza $1 \mathrm{~m}^{2}$ & $0+1$ & 4 & 68,5 & $\mathrm{~b}$ & $\mathrm{C}$ & 2,9 & $\mathrm{~b}$ & $\mathrm{C}$ & 4 & 83 & 38,3 & $\mathrm{~b}$ & $\mathrm{C}$ & 2,9 & $\mathrm{~b}$ & $\mathrm{C}$ \\
\hline & & $0+1+2$ & 4 & 73,8 & $\mathrm{a}$ & & 3,1 & $\mathrm{a}$ & & 4 & 88 & 41,5 & $\mathrm{a}$ & & 3,1 & $\mathrm{a}$ & \\
\hline & Sin control & Sin control & 4 & 68,9 & & & 2,8 & & $\mathrm{C}$ & 4 & 81 & 37,2 & & $\mathrm{D}$ & 2,8 & $\mathrm{C}$ & \\
\hline & & 0 & 4 & 97,3 & $\mathrm{~b}$ & & 3,9 & $\mathrm{~b}$ & & 4 & 96 & 55,7 & $\mathrm{~b}$ & & 3,9 & $\mathrm{~b}$ & \\
\hline & Total & $0+1$ & 4 & 119,5 & $\mathrm{~b}$ & A & 4,21 & $a b$ & B & 4 & 99 & 65,3 & $\mathrm{~b}$ & B & 4,2 & $a b$ & \\
\hline Etiopía (Rojo & & $0+1+2$ & 4 & 145,3 & $\mathrm{a}$ & & 4,6 & $\mathrm{a}$ & & 4 & 90 & 79,3 & $\mathrm{a}$ & & 4,6 & $\mathrm{a}$ & \\
\hline arcilloso) & & 0 & 4 & 115,2 & $3 \mathrm{~b}$ & & 4,7 & $\mathrm{~b}$ & & 4 & 100 & 73,1 & $\mathrm{~b}$ & & 4,7 & $\mathrm{~b}$ & \\
\hline & Banda $2 \mathrm{~m}$ & $0+1$ & 4 & 121,6 & $\mathrm{~b}$ & A & 4,8 & $a b$ & $\mathrm{~A}$ & 4 & 99 & 75,3 & $\mathrm{~b}$ & A & 4,8 & $a b$ & \\
\hline (1998-2002) & & $0+1+2$ & 4 & 128,8 & $\mathrm{a}$ & & 4,9 & $\mathrm{a}$ & & 4 & 100 & 80,5 & $\mathrm{a}$ & & 4,9 & $\mathrm{a}$ & \\
\hline & & 0 & 4 & 104,1 & $\mathrm{~b}$ & & 4,1 & $\mathrm{~b}$ & & 4 & 99 & 60,6 & $\mathrm{~b}$ & & 4,1 & $\mathrm{~b}$ & \\
\hline & Taza $1 \mathrm{~m}^{2}$ & $0+1$ & 4 & 101,2 & $\mathrm{~b}$ & B & 3,9 & $\mathrm{~b}$ & B & 4 & 97 & 57,4 & $\mathrm{~b}$ & $\mathrm{C}$ & 3,9 & $\mathrm{~b}$ & \\
\hline & & $0+1+2$ & 4 & 106,4 & a & & 4,1 & $\mathrm{a}$ & & 4 & 99 & 62,8 & $\mathrm{a}$ & & 4,1 & $\mathrm{a}$ & \\
\hline & Sin control & Sin control & 2 & 32,6 & & $\mathrm{C}$ & 1,3 & & $\mathrm{C}$ & 4 & 96 & 75,9 & & $\mathrm{C}$ & 4,4 & $\mathrm{C}$ & \\
\hline & & 0 & 2 & 53 & $\mathrm{a}$ & & 1,9 & $\mathrm{a}$ & & 4 & 98 & 104,2 & $\mathrm{a}$ & & 5,4 & $\mathrm{a}$ & \\
\hline Entrecaminos & Total & $0+1$ & 2 & 53 & $\mathrm{a}$ & A & 1,9 & $\mathrm{a}$ & A & 4 & 96 & 105,2 & $\mathrm{a}$ & A & 5,4 & & \\
\hline (Cenizas & & $0+1+2$ & 2 & 48,6 & $\mathrm{a}$ & & 1,8 & $\mathrm{a}$ & & 4 & 90 & 101,9 & $\mathrm{a}$ & & 5,3 & $\mathrm{a}$ & \\
\hline volcánicas & & 0 & 2 & 49 & $\mathrm{a}$ & & 1,8 & $\mathrm{a}$ & & 4 & 97 & 100,7 & $\mathrm{a}$ & & 5,3 & $\mathrm{a}$ & \\
\hline recientes) & Banda $2 \mathrm{~m}$ & $0+1$ & 2 & 53 & $\mathrm{a}$ & A & 1,9 & $\mathrm{a}$ & $\mathrm{A}$ & 4 & 98 & 101,6 & $\mathrm{a}$ & A & 5,3 & & $A B$ \\
\hline & & $0+1+2$ & 2 & 53 & $\mathrm{a}$ & & 1,9 & $\mathrm{a}$ & & 4 & 96 & 104,7 & $\mathrm{a}$ & & 5,2 & $\mathrm{a}$ & \\
\hline (1998-2000) & & 0 & 2 & 42,6 & $\mathrm{a}$ & & 1,7 & $\mathrm{a}$ & & 4 & 94 & 92,8 & $\mathrm{a}$ & & 5,2 & $\mathrm{a}$ & \\
\hline & Taza $1 \mathrm{~m}^{2}$ & $0+1$ & 2 & 43,3 & a & B & 1,6 & $\mathrm{a}$ & B & 4 & 94 & 88,9 & $\mathrm{a}$ & B & 4,9 & $\mathrm{a}$ & \\
\hline & & $0+1+2$ & 2 & 42,3 & a & & 1,6 & $\mathrm{a}$ & & 4 & 91 & 90,1 & $\mathrm{a}$ & & 4,9 & $\mathrm{a}$ & \\
\hline
\end{tabular}

Letras distintas denotan diferencias significativas según pruebas de Tukey $(\mathrm{p}<0,05)$. Letras mayúsculas y minúsculas indican diferencias entre Tratamientos o Intensidades y Subtratamientos o Periodicidades, respectivamente. 
BOSQUE 25(2): 57-67, 2004

Impacto de la duración y geometría del control de malezas en la productividad de plantaciones...

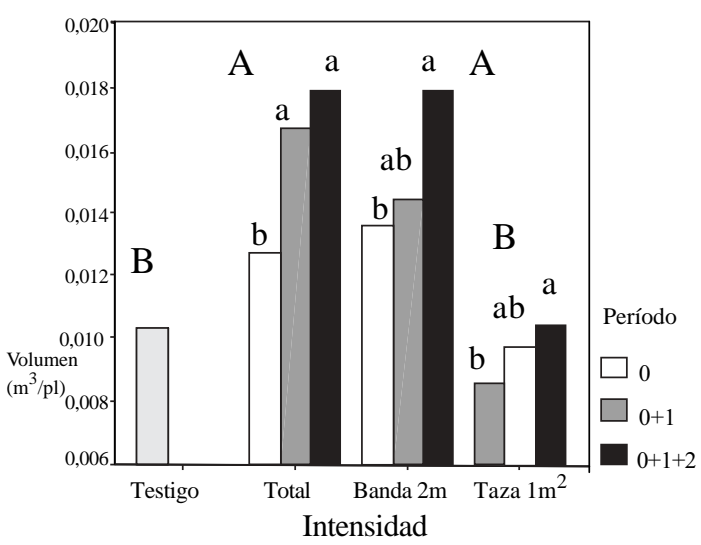

Figura 1. Volumen (m³/planta) en sitio Escuadrón (2002). Edad: 6 años.

Volume (cubic meter/tree) in Escuadrón site (2002). Age: 6.

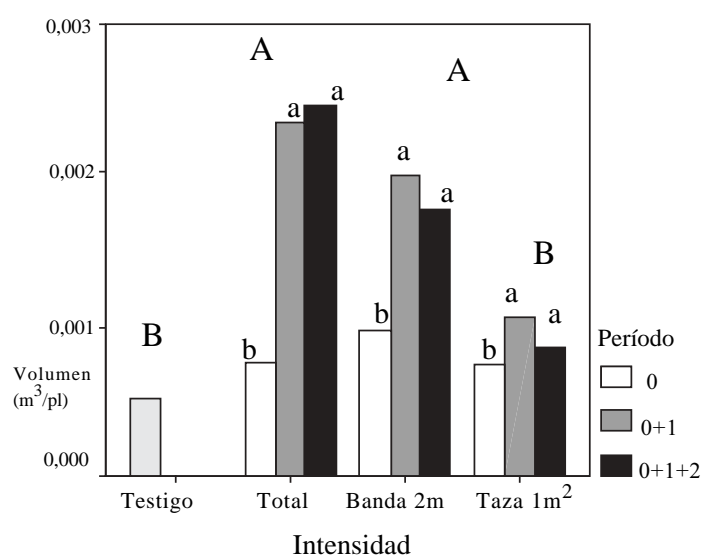

Figura 2. Volumen (m³/planta) en sitio San Pedro (2001). Edad: 5 años.

Volume (cubic meter/tree) in San Pedro site (2001). Age: 5.

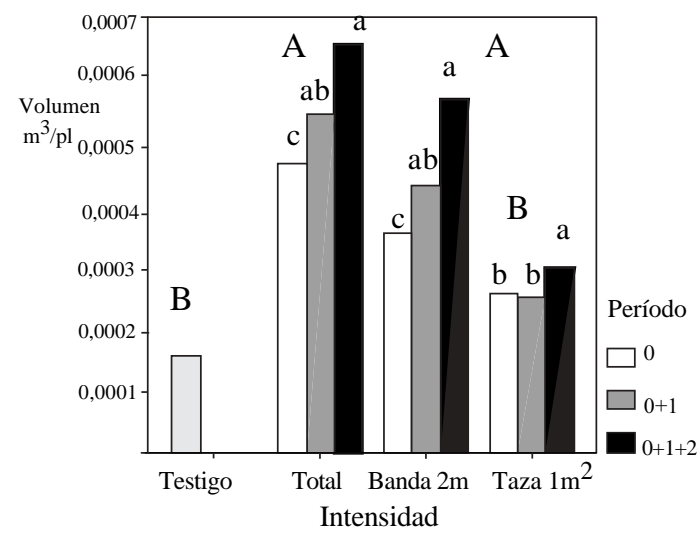

Figura 3. Volumen $\left(\mathrm{m}^{3} / \mathrm{pl}\right)$ en fundo Coihueco (2002). Edad: 4 años.

Volume (cubic meter/tree) in Coihueco site (2002). Age: 4.
Al analizar lo sucedido con la periodicidad en los sitios Escuadrón y Coihueco (figuras 1 y 3), se observa que aplicar por 3 años es levemente mejor que aplicar sólo por 2, en cambio en San Pedro (figura 2) no hay diferencias en controlar malezas por 2 ó 3 años. En general, la significativa superioridad estadística $(\mathrm{p}<0,05)$ de controlar por 2 ó 3 años respecto de aplicar sólo por 1 , se debe, según Sands (7), a que los árboles están más propensos a estrés en su primer verano después de plantación porque tienen un sistema de raíces más superficial y no pueden competir por el agua con las raíces de las malezas. Así, el control de malezas es más crítico en el período inicial de la plantación, aun cuando la densidad de cobertura de malezas pueda incrementar en los años posteriores.

En el sitio Etiopía (figura 4) se observa que la mejor periodicidad es por 3 años con el control en bandas. En el sitio Entrecaminos (figura 5) no hay diferencias entre aplicar control total o en bandas en cualquiera de las tres periodicidades, lo que quizás se deba al tipo de suelo de esta zona (alta retención de humedad). Al respecto, en estudios de Intensidad de control de malezas en sitios de la IX Región, Izquierdo (2), concluye que el tratamiento en bandas en plantaciones establecidas en suelos fértiles es altamente conveniente.

Es importante destacar que en los sitios Escuadrón y San Pedro se evidencia una interacción significativa $(\mathrm{p}<0,05)$ entre la intensidad y periodicidad de control de malezas, lo cual es válido para el DAC, altura y volumen (anexo 2).

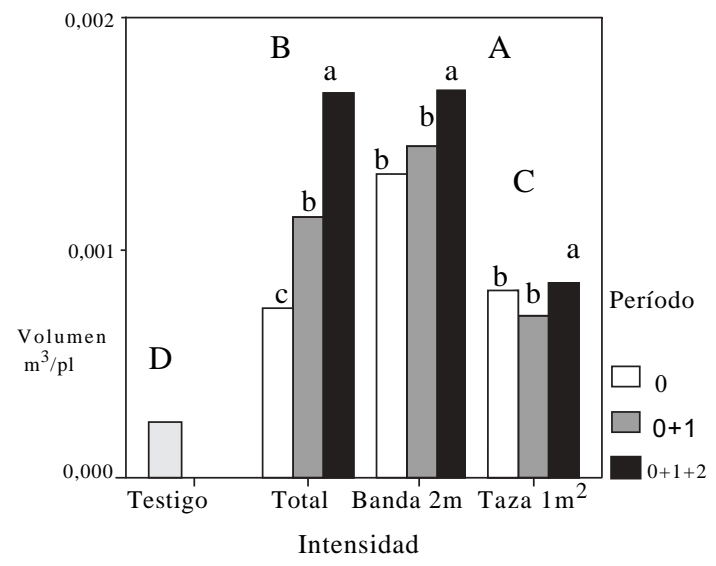

Figura 4. Volumen (m³/planta) en sitio Etiopía (2002). Edad: 4 años.

Volume (cubic meter/tree) in Etiopía site (2002). Age: 4. 


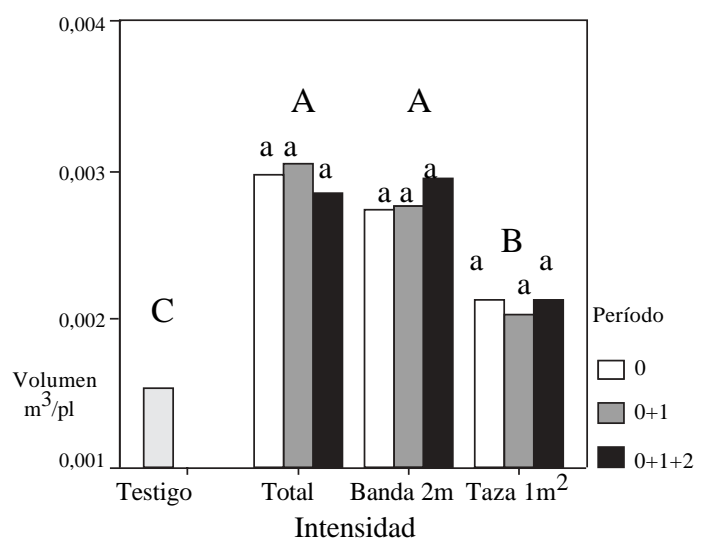

Figura 5. Volumen ( $\mathrm{m}^{3} /$ planta) en sitio Entrecaminos (2002). Edad: 4 años.

Volume (cubic meter/tree) in Entrecaminos site (2002). Age: 4.
Los principales resultados del análisis económico se presentan en cuadro 3.

Para el sitio metamórfico costero, la TIR (tasa interna de retorno) diferencial varía entre $11,3 \%$ y $14,1 \%$, sugiriendo que un control óptimo sería del total los años $0+1$. No obstante, desde una perspectiva más racional, debería tenderse a aplicar un control en banda de $2 \mathrm{~m}$ de ancho el año $0 \mathrm{y}$ un control total el año 1. La menor exploración radicular de la especie durante el año 0 , la baja demanda de agua y la adecuada capacidad de retención de humedad del suelo, permitiría soportar el control sólo en parte de la superficie. Por otra parte, para el año 1, la mayor demanda hídrica de las plantas, exigiría una drástica reducción de la vegetación competidora.

\section{CUADRO 3}

Ganancia por tratamientos de duración y geometría de control de malezas.

Gain according to duration and geometry of weed control.

\begin{tabular}{|c|c|c|c|c|c|c|c|c|}
\hline Fundo/Sitio & $\begin{array}{l}\text { Tratamiento } \\
\text { (Intensidad) }\end{array}$ & $\begin{array}{c}\text { Subtratamiento } \\
\text { (Periodicidad) }\end{array}$ & $\begin{array}{c}\text { Ganancia } \\
\text { Área Basal } \\
\text { (6 años) }\end{array}$ & $\begin{array}{l}\text { Ganancia } \\
\text { Altura media } \\
\text { (6 años) }\end{array}$ & $\begin{array}{l}\text { Ganancia } \\
\text { volumen } \\
(20 \text { años })\end{array}$ & Manejo & $\begin{array}{l}\text { Edad } \\
\text { corta } \\
\text { (años) }\end{array}$ & $\begin{array}{c}\text { TIR } \\
\text { Tratamiento } \\
(\%)\end{array}$ \\
\hline \multirow{6}{*}{$\begin{array}{l}\text { Escuadrón } \\
\text { (Metamórfico } \\
\text { costero) }\end{array}$} & Total & 0 & 3,3 & 0,7 & 48 & \multirow{6}{*}{ Intensivo } & \multirow{6}{*}{25} & 12,0 \\
\hline & & $0+1$ & 8,0 & 1,4 & 128 & & & 14,1 \\
\hline & & $0+1+2$ & 10,3 & 1,7 & 173 & & & 13,7 \\
\hline & Banda $2 \mathrm{~m}$ & 0 & 4,3 & 0,9 & 71 & & & 13,2 \\
\hline & & $0+1$ & 4,9 & 1,3 & 78 & & & 11,3 \\
\hline & & $0+1+2$ & 9,5 & 1,9 & 148 & & & 12,9 \\
\hline \multirow{6}{*}{$\begin{array}{l}\text { San Pedro } \\
\text { (Granítico) }\end{array}$} & Total & 0 & 0,5 & 0,6 & 11 & \multirow{6}{*}{$\begin{array}{l}\text { Multi- } \\
\text { propósito }\end{array}$} & \multirow{6}{*}{25} & 4,7 \\
\hline & & $0+1$ & 2,8 & 0,5 & 47 & & & 8,2 \\
\hline & & $0+1+2$ & 2,8 & 0,3 & 46 & & & 6,4 \\
\hline & Banda $2 \mathrm{~m}$ & 0 & 1,1 & 0,8 & 24 & & & 6,9 \\
\hline & & $0+1$ & 3,0 & 0,7 & 54 & & & 8,4 \\
\hline & & $0+1+2$ & 2,4 & 0,6 & 46 & & & 6,4 \\
\hline \multirow{6}{*}{$\begin{array}{c}\text { Coihueco } \\
\text { (Arena) }\end{array}$} & Total & 0 & 2,3 & 0,1 & 29 & \multirow{6}{*}{ Pulpable } & \multirow{6}{*}{20} & 9,6 \\
\hline & & $0+1$ & 2,7 & 0,2 & 33 & & & 6,6 \\
\hline & & $0+1+2$ & 3,1 & 0,3 & 38 & & & 5,4 \\
\hline & Banda $2 \mathrm{~m}$ & 0 & 1,5 & 0,0 & 16 & & & 3,5 \\
\hline & & $0+1$ & 2,0 & 0,0 & 25 & & & 5,0 \\
\hline & & $0+1+2$ & 2,7 & 0,2 & 33 & & & 4,7 \\
\hline \multirow{6}{*}{$\begin{array}{c}\text { Etiopía } \\
\text { (Rojo } \\
\text { arcilloso) }\end{array}$} & Total & 0 & 2,7 & 1,5 & 61 & \multirow{6}{*}{$\begin{array}{l}\text { Multi- } \\
\text { propósito }\end{array}$} & \multirow{6}{*}{25} & 13,2 \\
\hline & & $0+1$ & 4,0 & 2,1 & 84 & & & 11,5 \\
\hline & & $0+1+2$ & 6,6 & 2,9 & 127 & & & 12,4 \\
\hline & Banda $2 \mathrm{~m}$ & 0 & 5,2 & 2,4 & 101 & & & 15,0 \\
\hline & & $0+1$ & 5,5 & 2,4 & 106 & & & 12,8 \\
\hline & & $0+1+2$ & 6,3 & 2,5 & 116 & & & 11,8 \\
\hline \multirow{6}{*}{$\begin{array}{c}\text { Entrecaminos } \\
\text { (Cenizas } \\
\text { volcánicas } \\
\text { recientes) }\end{array}$} & Total & 0 & 5,3 & 0,4 & 69 & \multirow{6}{*}{ Intensivo } & \multirow{6}{*}{25} & 14,0 \\
\hline & & $0+1$ & 5,4 & 0,3 & 67 & & & 10,5 \\
\hline & & $0+1+2$ & 4,9 & 0,6 & 67 & & & 8,8 \\
\hline & Banda $2 \mathrm{~m}$ & 0 & 5,0 & 1,5 & 87 & & & 13,6 \\
\hline & & $0+1$ & 4,7 & 0,6 & 63 & & & 9,8 \\
\hline & & $0+1+2$ & 5,7 & 1,0 & 86 & & & 9,8 \\
\hline
\end{tabular}


BOSQUE 25(2): 57-67, 2004

Impacto de la duración y geometría del control de malezas en la productividad de plantaciones...

El sitio granítico del secano interior de la cordillera de Nahuelbuta (sector de la "sombra de lluvia") entrega una TIR entre $4,7 \%$ a $8,4 \%$. A pesar del severo estrés hídrico estival del sitio, resulta económicamente aconsejable dos controles en banda de $2 \mathrm{~m}$ de ancho. Este ensayo sufrió algunos daños por animales, lo que puede haber afectado el crecimiento de las plantas.

Para el sitio de arenas, la TIR varía entre 3,5\% a $9,6 \%$, siendo aconsejable aplicar un solo control total de maleza el año 0. Dado que el destino de esta plantación sería madera pulpable, no permite soportar un mayor nivel de inversión.

En el sitio rojo arcilloso del Valle Central la TIR de los tratamientos evaluados varía entre $11,5 \%$ a $15 \%$, siendo el óptimo el control en banda de $2 \mathrm{~m}$ de ancho por sólo una vez. Este ensayo, instalado el año 1998, durante el cual en el área se midieron $598 \mathrm{~mm}$ de precipitación versus 1.354 mm en un año normal (13), puede haber favorecido exageradamente el control en banda del año 0 , en desmedro del control al año 1. Por lo anterior, la estrategia más racional sería la de aplicar dos controles en banda de $2 \mathrm{~m}$ de ancho (años 0 y 1 ).

Para el sitio de cenizas volcánicas recientes, la TIR varía entre $8,8 \%$ a $14 \%$. En esta condición, donde la mayor competencia la presentan malezas de tipo herbáceas, se sugiere aplicar dos controles en banda de $2 \mathrm{~m}$ de ancho. Una estrategia más intensiva sería una similar a la sugerida al sitio metamórfico costero; no obstante, dada la gran frecuencia de "speed-wobble" o deformación fustal, tema reportado inicialmente en Australia por Carlyle (17), es recomendable dejar un área cubierta de malezas, las que puedan capturar el exceso de nitrógeno disponible al inicio del desarrollo de la plantación.

Otras combinaciones de tratamientos no fueron evaluadas, por lo cual se están instalando algunos ensayos. Las opciones a evaluar son de banda $2 \mathrm{~m}$ de ancho + total los años 1 y 2 . Esta estrategia se sugiere para los sitios de mayor productividad, tales como sedimentos marinos, metamórficos costeros y cenizas volcánicas recientes.

Durante el desarrollo del estudio se ha detectado una carencia en cuanto a la ecofisiología de las malezas principales en la zona centro-sur del país. Un mejor conocimiento acerca del despliegue foliar de las malezas, en función de variables climáticas, permitiría precisar la oportunidad de control. Asi- mismo, permitiría apoyar el desarrollo de modelos de optimización que incluyan una visión continua del efecto de las malezas en los cultivos.

Al momento de iniciar el estudio (1996), no fue posible disponer, a un costo razonable, de una batería de sensores para medir el contenido de humedad del suelo, lo que habría permitido precisar el impacto de las malezas en la competencia del recurso agua. Además, la información disponible a partir de este tipo de estudios permitiría alimentar futuros modelos de apoyo a la toma de decisiones (18).

\section{CONCLUSIONES}

- En general, los mejores tratamientos para las variables DAC y altura son el control de malezas total (T2) y el control de malezas en banda de $2 \mathrm{~m}$ de ancho (T3), lo que se cumple a cabalidad con la variable volumen, excepto en el sitio Etiopía, donde el control en banda fue superior.

- El control de malezas en tazas de 1 metro cuadrado no es recomendable para ningún ecosistema debido a que en la mayoría de los ensayos es similar o levemente superior al testigo sin control de malezas.

- En sitios de arenas secas, a pesar de ser biológicamente recomendable aplicar más de un control de malezas, un análisis económico restringe seriamente esta posibilidad a sólo un control total al año 0 .

- En cuanto a la periodicidad por tratamiento, en el sitio metamórfico costero, se recomienda aplicar por los dos primeros años de establecida la plantación, el primero con un control en banda de $2 \mathrm{~m}$ de ancho y el segundo en una superficie mayor.

- En los sitios rojo arcilloso y graníticos, la periodicidad recomendada es $0+1$ en banda de 2 $\mathrm{m}$ de ancho.

- En el sitio de cenizas volcánicas recientes, la estrategia de control en banda de $2 \mathrm{~m}$ de ancho por dos períodos es la más conveniente. 


\section{BIBLIOGRAFIA}

(1) KOGAN, M., R. FIGUEROA. Interferencia producida por las malezas durante los dos primeros años en Pinus radiata D. Don. Bosque (Chile), 1999, vol. 20, No 1, p. 57-64.

(2) IZQUIERDO, H. Control de malezas herbáceas durante el primer año de establecimiento de Pinus radiata D. Don en tres sitios de la IX Región. Tesis Ingeniero Forestal, Facultad de Ciencias Forestales, Universidad Austral de Chile, Valdivia, Chile. 1995. 94 p.

(3) ADAMS, P.R., G.W. DUTKOWSKI. Response of radiata pine to pattern of weed control in South Australia. In: Gaskin. R.E. and Zabkiewicz J.A. New Zealand. 20-24, 1995. FRI Bulletin No 192, pp. 179-181.

(4) ADAMS, P.R., C.L. BEADLE, N.J. MENDHAM, P.J. SMETHURST. The impact of timing and duration of grass control on growth of a young Eucalyptus globulus Labill. Plantation. New Forest. 2003, vol. 26, p. 147-165.

(5) MARSICO, O.J.V. Herbicidas y fundamentos del control de malezas. $1^{\mathrm{a}}$ edición. Editorial Hemisferio Sur. Buenos Aires. Argentina. 1980. 120 p.

(6) MONTENEGRO, P. Efecto del período y cobertura del control de malezas en el desarrollo de plantaciones de Pinus taeda L. Tesis Ing. Agrónomo, Universidad del Salvador, Sta. Cruz, Argentina, 1998. 110 p.

(7) SANDS, R., E.K.S. NAMBIAR. Water relations of Pinus radiata in competition with weeds. Canadian Journal of Forestry Research. 1984, vol. 14, p. 233-237.

(8) BOOMSMA, D.B., I.R. HUNTER. Effects of water, nutrients and their interactions on tree growth, and plantation forest management practices in Australasia: A review. For. Ecol. Manage. 1990, vol. 30, p. 455-476.

(9) SMETHURST, P.J., E.K.S. NAMBIAR. Role of weeds in the management of nitrogen in a young Pinus radiata plantation. New Forests. 1989, vol. 3, p. 203-224.

(10) ÖRLANDER, G., U. NILSSON AND J. HÄLLGREN. Competition for water and nutrients between ground vegetation and planted Picea abies. New Zealand Journal of Forestry Science. 1996, vol. 26, p. 99-117.

(11) MACLAREN, P. Radiata pine grower's manual. Chemical weed control. New Zealand, 1993, p. 34-39. (FRI N. 184)

(12) BISOGNIN, E. Efeito de cobertura e períodos de manejo de plantas daninhas no desenvolvimento inicial de Pinus elliottii, pinus taeda e Pinus elliottii var. elliottii x Pinus caribaea var. hondurensis em Várzeas. 2002. Tesis Magister, Universidad Federal de Santa María, RS-Brasil. $105 \mathrm{p}$.

(13) SANTIBAÑEZ, F. Atlas agroclimático de Chile. VI, VII, VIII y IX Regiones. Laboratorio de Agroclimatología, Facultad de Ciencias Agrarias y Forestales, Universidad de Chile. Ministerio de Agricultura, Fondo de Investigación Agropecuaria, Corporación de Fomento de la Producción. Santiago. 1993. 73 p.

(14) STEEL, R.G. Y J.H. TORRIE. Bioestadística: Principios y Procedimientos. McGraw-Hill Latinoamericana S. A 1985. 622 p.

(15) SNOWDON, P., H.D. WARING. Long-term nature of growth responses obtained to fertiliser and weed control applied at planting and their consequences for forest management, In: Proceedings of the IUFRO symposium on site and site productivity of fast growing plantations. Pretoria and Petermaritzberg, South Africa, 1984, 701-711.

(16) RICHARDSON, B., M. KIMBERLEY, A. PATTISON. Pinus radiata growth benefits from spot weed control in Kinleith Forest. Forest Weeds and Pests. Proceedings 50th New Zealand. Plant Protection Conference. 1997, p. 369-372.

(17) CARLYLE, J.C., N.D. TURVEY, P. HOPMANS, G.M. DOWNES. Stem deformation in Pinus radiata associated with previous land use. Canadian Journal of Forest Research. 1989, vol. 19, $\mathrm{N}^{\mathrm{o}} 1$, p. 96-105.

(18) ALVAREZ, J. Diseño, construcción y evaluación de un modelo de apoyo a la toma de decisiones en control químico de malezas, en plantaciones de Pinus radiata D. Don. Tesis MSc Economía Agraria. Pontificia Universidad Católica de Chile. 2002. 94 p. 
BOSQUE 25(2): 57-67, 2004

Impacto de la duración y geometría del control de malezas en la productividad de plantaciones...

\section{ANEXO 1}

Descripción por ensayo del tipo de suelo y clima.

Description of soil and climate of trial sites.

\begin{tabular}{|c|c|c|}
\hline Fundo & Coordenadas & Clima \\
\hline San Pedro & $37^{\circ} 35^{\prime}, 72^{\circ} 39^{\prime}$ & $\begin{array}{l}\text { Templado mesotermal estenotérmico Mediterráneo subhúmedo. } \\
\text { Temperaturas entre } 28,6 \text { (Enero) a } 4,4 \text { (Julio) }{ }^{\circ} \mathrm{C} \text {. Período libre } \\
\text { de heladas de } 235 \text { días ( } 9 \text { heladas por año). Precipitación anual } \\
\text { de } 1.093 \mathrm{~mm} \text {, déficit hídrico de } 716 \mathrm{~mm} \text { y } 5 \text { meses de período } \\
\text { seco. }\end{array}$ \\
\hline Escuadrón & $36^{\circ} 56^{\prime}, 73^{\circ} 8^{\prime}$ & $\begin{array}{l}\text { Templado mesotermal inferior estenotérmico-mediterráneo } \\
\text { subhúmedo. Temperaturas entre } 23,2 \text { (Enero) a } 6,0 \text { (Julio) }{ }^{\circ} \mathrm{C} \text {. } \\
\text { Período libre de heladas de } 322 \text { días ( } 1 \text { helada por año). Precipi- } \\
\text { tación anual de } 1.437 \mathrm{~mm} \text {, déficit hídrico de } 449 \mathrm{~mm} \text { y } 5 \text { meses } \\
\text { de período seco. }\end{array}$ \\
\hline Etiopía & $37^{\circ} 54^{\prime}, 72^{\circ} 22^{\prime}$ & $\begin{array}{l}\text { Templado mesotermal inferior estenotérmico-mediterráneo } \\
\text { subhúmedo. Temperaturas entre } 25,7 \text { (Enero) a } 4,1 \text { (Julio) }{ }^{\circ} \mathrm{C} \text {. } \\
\text { Período libre de heladas de } 213 \text { días ( } 13 \text { heladas por año). Pre- } \\
\text { cipitación anual de } 1.354 \mathrm{~mm} \text {, déficit hídrico de } 516 \mathrm{~mm} \text { y } 5 \\
\text { meses de período seco. }\end{array}$ \\
\hline Coihueco & $37^{\circ} 28^{\prime}, 72^{\circ} 3^{\prime}$ & $\begin{array}{l}\text { Templado mesotermal inferior estenotérmico-mediterráneo } \\
\text { semiárido. Temperaturas entre } 30,3 \text { (Enero) a } 4,3 \text { (Julio) }{ }^{\circ} \mathrm{C} \text {. } \\
\text { Período libre de heladas de } 230 \text { días ( } 11 \text { heladas por año). Pre- } \\
\text { cipitación anual de } 917 \mathrm{~mm} \text {, déficit hídrico de } 819 \mathrm{~mm} \text { y } 6 \\
\text { meses de período seco. }\end{array}$ \\
\hline Entrecaminos & $38^{\circ} 28^{\prime}, 72^{\circ} 13^{\prime}$ & $\begin{array}{l}\text { Templado mesotermal inferior estenotérmico-mediterráneo } \\
\text { subhúmedo. Temperaturas entre } 24,3 \text { (Enero) a } 3,9 \text { (Julio) }{ }^{\circ} \mathrm{C} \text {. } \\
\text { Período libre de heladas de } 193 \text { días ( } 18 \text { heladas por año). Pre- } \\
\text { cipitación anual de } 1.389 \mathrm{~mm} \text {, déficit hídrico de } 416 \mathrm{~mm} \text { y } 5 \\
\text { meses de período seco. }\end{array}$ \\
\hline
\end{tabular}


BOSQUE 25(2): 57-67, 2004

Impacto de la duración y geometría del control de malezas en la productividad de plantaciones...

\section{ANEXO 2}

Tablas de Análisis de varianza (probabilidad de mayores valores p) para Pinus radiata. Analysis of variance tables (probability of greater p-values) for Pinus radiata.

\begin{tabular}{|c|c|c|c|c|c|c|c|c|c|c|c|}
\hline & & \multicolumn{2}{|c|}{$\begin{array}{c}\text { Escuadrón } \\
\text { Edad: } 4 \text { años } \\
(1996-2000)\end{array}$} & \multicolumn{2}{|c|}{$\begin{array}{c}\text { San Pedro } \\
\text { Edad: } 4 \text { años } \\
(1996-2000)\end{array}$} & \multicolumn{2}{|c|}{$\begin{array}{c}\text { Coihueco } \\
\text { Edad: } 4 \text { años } \\
(1998-2002)\end{array}$} & \multicolumn{2}{|c|}{$\begin{array}{c}\text { Etiopía } \\
\text { Edad: } 4 \text { años } \\
(1998-2002)\end{array}$} & \multicolumn{2}{|c|}{$\begin{array}{c}\text { Entrecaminos } \\
\text { Edad: } 2 \text { años } \\
(1998-2000)\end{array}$} \\
\hline & & $\begin{array}{l}\text { DAC } \\
2000\end{array}$ & $\begin{array}{c}\text { Altura } \\
2000\end{array}$ & $\begin{array}{l}\text { DAC } \\
2000\end{array}$ & $\begin{array}{c}\text { Altura } \\
2000\end{array}$ & $\begin{array}{l}\text { DAC } \\
2002\end{array}$ & $\begin{array}{c}\text { Altura } \\
2002\end{array}$ & $\begin{array}{l}\text { DAC } \\
2002\end{array}$ & $\begin{array}{c}\text { Altura } \\
2002\end{array}$ & $\begin{array}{l}\text { DAC } \\
2000\end{array}$ & $\begin{array}{c}\text { Altura } \\
2000\end{array}$ \\
\hline \multicolumn{12}{|l|}{ Factor } \\
\hline Rep & 2 & 0,590 & 0,025 & 0,918 & 0,222 & 0,141 & 0,265 & 0,448 & 0,590 & 0,193 & 0,153 \\
\hline A & 3 & 0,001 & 0,000 & 0,000 & 0,001 & 0,000 & 0,002 & 0,005 & 0,007 & 0,001 & 0,001 \\
\hline Error (a) & 6 & & & & & & & & & & \\
\hline B & 2 & 0,000 & 0,012 & 0,007 & 0,141 & 0,000 & 0,000 & 0,000 & 0,017 & 0,331 & 0,183 \\
\hline $\mathrm{AxB}$ & 6 & 0,000 & 0,025 & 0,000 & 0,015 & 0,343 & 0,074 & 0,002 & 0,267 & 0,027 & 0,190 \\
\hline Error & 16 & & & & & & & & & & \\
\hline
\end{tabular}

Nivel de significancia : 0,05

A. Intensidad

B. Periodicidad

\begin{tabular}{|c|c|c|c|c|c|c|c|c|c|c|c|c|c|c|c|c|}
\hline & & \multicolumn{3}{|c|}{$\begin{array}{l}\text { Escuadrón } \\
\text { Edad: } 6 \text { años } \\
(1992-2002)\end{array}$} & \multicolumn{3}{|c|}{$\begin{array}{c}\text { San Pedro } \\
\text { Edad: } 5 \text { años } \\
(1996-2001)\end{array}$} & \multicolumn{3}{|c|}{$\begin{array}{c}\text { Coihueco } \\
\text { Edad: } 4 \text { años } \\
(1998-2002)\end{array}$} & \multicolumn{3}{|c|}{$\begin{array}{c}\text { Etiopía } \\
\text { Edad: } 4 \text { años } \\
\text { (1998-2002) }\end{array}$} & \multicolumn{3}{|c|}{$\begin{array}{c}\text { Entrecaminos } \\
\text { Edad: } 4 \text { años } \\
(1998-2002)\end{array}$} \\
\hline & & $\begin{array}{l}\text { DAP } \\
2002\end{array}$ & $\begin{array}{l}\text { Altura } \\
2002\end{array}$ & $\begin{array}{c}\text { Volumen } \\
2002\end{array}$ & $\begin{array}{l}\text { DAP } \\
2001\end{array}$ & $\begin{array}{c}\text { Altura } \\
2001\end{array}$ & $\begin{array}{c}\text { Volumen } \\
2001\end{array}$ & $\begin{array}{l}\text { DAP } \\
2002\end{array}$ & $\begin{array}{c}\text { Altura } \\
2002\end{array}$ & $\begin{array}{c}\text { Volumen } \\
2002\end{array}$ & $\begin{array}{l}\text { DAP } \\
2002\end{array}$ & $\begin{array}{c}\text { Altura } \\
2002\end{array}$ & $\begin{array}{c}\text { Volumen } \\
2002\end{array}$ & $\begin{array}{l}\text { DAP } \\
2002\end{array}$ & $\begin{array}{c}\text { Altura } \\
2002\end{array}$ & $\begin{array}{c}\text { Volumen } \\
2002\end{array}$ \\
\hline Factor & $\mathrm{df}$ & & & & & & & & & & & & & & & \\
\hline Rep & 2 & 0,223 & 0,025 & 0,021 & 0,629 & 0,433 & 0,308 & 0,225 & 0,265 & 0,712 & 0,620 & 0,590 & 0,486 & 0,834 & 0,362 & 0,743 \\
\hline $\begin{array}{l}\text { A } \\
\text { error (a) }\end{array}$ & $\begin{array}{l}3 \\
6\end{array}$ & 0,001 & 0,000 & 0,000 & 0,013 & 0,014 & 0,001 & 0,001 & 0,002 & 0,010 & 0,021 & 0,007 & 0,030 & 0,003 & 0,006 & 0,002 \\
\hline B & 2 & 0,004 & 0,001 & 0,003 & 0,004 & 0,009 & 0,008 & 0,000 & 0,000 & 0,016 & 0,000 & 0,017 & 0,001 & 0,626 & 0,569 & 0,448 \\
\hline $\begin{array}{l}\text { AxB } \\
\text { error }\end{array}$ & $\begin{array}{r}6 \\
16\end{array}$ & 0,408 & 0,348 & 0,140 & 0,161 & 0,337 & 0,016 & 0,264 & 0,074 & 0,251 & 0,023 & 0,267 & 0,023 & 0,717 & 0,918 & 0,553 \\
\hline
\end{tabular}

Nivel de significancia: 0,05

A. Intensidad

B. Periodicidad 